\title{
QUASITRIANGULAR MATRICES
}

\author{
J. DOMBROWSKI
}

ABSTRACT. It is shown that there exist quasitriangular operators which cannot be represented as quasitriangular matrices.

Introduction. A quasitriangular matrix is an infinite matrix $A=\left[a_{i j}\right]$ in which all entries below the subdiagonal are zero and the subdiagonal entries cluster at zero (i.e., $a_{i j}=0$ for $i>j+1$ and $\lim \inf \left|a_{j+1, j}\right|=0$ ). A quasitriangular operator is an operator which can be expressed as the sum of a triangular matrix $\left(a_{i j}=0\right.$ for $\left.i>j\right)$ and a compact one. The relationship between quasitriangular matrices and quasitriangular operators, and the significance of studying that relationship in conjunction with the invariant subspace problem, are discussed by Halmos in [2]. It is shown in [2] that every bounded quasitriangular matrix defines a quasitriangular operator. Halmos then asks whether every cyclic quasitriangular operator has a quasitriangular matrix. It will be shown below that the answer is no. A few preliminary ideas are needed.

Let $A=\int \eta d E_{\eta}$ be a bounded selfadjoint operator defined on a separable Hilbert space $\mathcal{H}$. By Weyl's theorem $A$ is the sum of a diagonal operator and a compact one. Hence $A$ is quasitriangular. Denote by $\mathcal{K}_{a}(A)$ the set of elements $x$ in $\mathcal{H}$ for which $\left\|E_{\eta} x\right\|^{2}$ is an absolutely continuous function of $\eta$. The subspace $\mathcal{H}_{a}(A)$ reduces $A\left[1\right.$, p. 104] and the restriction of $A$ to $\mathcal{H}_{a}(A)$ is called the absolutely continuous part of $A$. A result due to Kato [3] and Rosenblum [4] asserts that the absolutely continuous part of the operator $A$ remains stable under a trace class perturbation. In particular, if $C$ is selfadjoint and of trace class, and if $B=A+C$, then the absolutely continuous parts of $A$ and $B$ are unitarily equivalent.

Main result. The main result to be established is as follows.

Proposition. A selfadjoint operator with a nontrivial absolutely continuous part cannot be represented as a quasitriangular matrix.

Proof. Let $A$ be a selfadjoint operator with a nontrivial absolutely continuous part. Suppose that with respect to some orthonormal basis, $A$ can be represented as a quasitriangular matrix. Clearly this matrix takes the form

Received by the editors August 22, 1977.

AMS (MOS) subject classifications (1970). Primary 47B15.

C. American Mathematical Society 1978 


$$
\left[\begin{array}{cccccc}
b_{1} & a_{1} & 0 & 0 & 0 & \ldots \\
a_{1} & b_{2} & a_{2} & 0 & 0 & \ldots \\
0 & a_{2} & b_{3} & a_{3} & 0 & \cdots \\
0 & 0 & a_{3} & b_{4} & a_{4} & \cdots \\
\vdots & \vdots & \vdots & \vdots & \vdots &
\end{array}\right]
$$

with some subsequence of $\left\{a_{n}\right\}$ converging to zero. Furthermore, the subsequence $\left\{a_{n_{k}}\right\}$ can be chosen so that $\Sigma\left|a_{n_{k}}\right|<\infty$.

Let $B$ be the matrix obtained from $A$ by replacing each $a_{n_{k}}$ by zero. Then $B$ has finite dimensional invariant subspaces. In fact, $B$ has a pure point spectrum.

If $C=A-B$ then $C$ is the real part of a weighted shift with weight sequence $\left\{c_{n}\right\}$ satisfying $\Sigma\left|c_{n}\right|<\infty$. Hence $C$ is of trace class. Since $A$ has an absolutely continuous part it follows, from the Kato-Rosenblum theorem, that $A-C=B$ has an absolutely continuous part. But this contradicts the fact that $B$ has a pure point spectrum.

COROLlaRY. The real part of the unilateral shift does not have a quasitriangular matrix.

\section{REFERENCES}

1. P. R. Halmos, Introduction to Hilbert space and the theory of spectral multiplicity, Chelsea, New York, 1951.

2. Some unsolved problems of unknown depth about operators on Hilbert space, Proc. Roy. Soc. Edinburgh Sect. A 76 (1976), 67-76.

3. T. Kato, Perturbation of continuous spectra by trace class operators, Proc. Japan Acad. 33 (1957), 260-264.

4. M. Rosenblum, Perturbation of the continuous spectrum and unitary equivalence, Pacific J. Math. 7 (1957), 997-1010.

Department of Mathematics, Wright State University, Dayton, Oho 45435 